\title{
Regression of Kaposi's Sarcoma after Anti- Tuberculous Therapy In A HIV-Negative Case With Disseminated Tuberculosis
}

\author{
Ko Chang1,2,3,4, Wei-Ru Lin ${ }^{1,2}$, Po-Chih Chen ${ }^{1,5,6}$ and Jih-Jin Tsai*1,2,3 \\ ${ }^{1}$ Tropical Medicine Center, Kaohsiung Medical University Hospital, Taiwan \\ ${ }^{2}$ Division of Infectious Diseases, Department of Internal Medicine, Kaohsiung Medical University Hospital, Taiwan \\ ${ }^{3}$ School of Medicine, College of Medicine, Kaohsiung Medical University, Kaohsiung, Taiwan \\ ${ }^{4}$ Department of Internal Medicine, Kaohsiung Municipal Siao-Kang Hospital, Kaohsiung, Taiwan \\ ${ }^{5}$ Department of Medical Laboratory Science and Biotechnology, College of Health Sciences, Kaohsiung Medical University, Taiwan \\ ${ }^{6}$ Department of Laboratory Medicine, Kaohsiung Medical University Hospital, Taiwan
}

Received: April 11, 2018; Published: May 17, 2018

*Corresponding author: Jih Jin Tsai, Division of Infectious diseases, Department of Internal Medicine, Kaohsiung Medical University Hospital, 100 Tzyou 1st Road, Kaohsiung 807, Taiwan

Abstract

Although Kaposi sarcoma occurs in immune compromised patients, including Human Immunodeficiency Virus (HIV) infection patients, Kaposi sarcoma associated with disseminated tuberculosis has rarely been reported on. We present a case of Kaposi sarcoma that developed in an HIV-negative patient with disseminated tuberculosis. The Kaposi sarcoma completely regressed with antituberculous therapy without the institution of any chemotherapy, radiotherapy or surgery. The patient remained disease-free after a long-term follow-up over five years.

\section{Case Report}

This 83 year-old male without systemic disease history, was hospitalized due to fever, cough, dyspnea, and swelling of right knee and right lower leg for one week. After admission, on October 11, 2008, Chest X-ray revealed fibrocalcified lesions over right lung with pleural effusion, and pneumonia subsequently progressed over the right lower lobe and left upper lobe within 2 weeks. Acidfast bacilli from the sputum was found and pulmonary tuberculosis (TB) was diagnosed. We also noted an ulcer of his upper lip, and the biopsy showed granulomatous tissue with ulcerative surface, many horse-shoe shaped multinucleated giant cells and positive acid-fast bacilli. Synovial fluid aspirated from the right knee examined for TB-PCR (polymerase chain reaction) turned out to be positive.

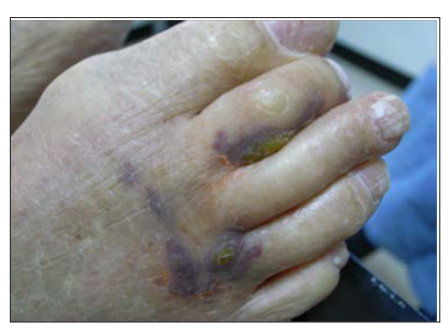

Figure 1a: Kaposi's sarcoma on the right foot after 7 days of anti-TB treatment.
We initiated anti-TB regimen of rifater (rifampicin $120 \mathrm{mg}$, isoniazid $80 \mathrm{mg}$, pyrazinamide $250 \mathrm{mg}$ ) at 4 tablets daily from October 15, 2008, and then 2 months later we shifted it to rifinah (rifampicin300 mg, isoniazid 150mg) at 2 tablets daily. However, the sputum and synovial fluid culture showed no growth of tuberculosis. At the same time, multiple indurated, painless, violaceous, nonpruritic skin lesions occurred on both feet and legs (Figure 1a), which was confirmed to be Kaposi's sarcoma (KS) via the histopathologic findings from the skin biopsy; the acid-fast stain failed to reveal any microorganism. The patient did not notice this skin lesion before and the patient's ELISA test of HIV showed negative. He had normal CD4/CD8 ratio (1.15) and normal absolute CD4 count Cytomegalovirus IgG, VZV and HSV IgG showed positive but HBsAg, anti-HCV Ab and HTLV 1/2 (human T-lymphotropic virus) showed negative result. Chemotherapy was not performed because of his initially poor general health condition. The KS lesions worsened after two months of TB treatment (Figure 1b), but unexpectedly regressed thereafter. A one-year treatment course for disseminated TB was completed. The KS lesions completely subsided and no relapse was detected after the 5- year regular follow-up (Figure 1c). His anemia was also improved with an increase of hemoglobin ( $\mathrm{Hb}$ ) from $8.4 \mathrm{~g} / \mathrm{dl}$ to $13.3 \mathrm{~g} / \mathrm{dL}$ (Figure 1c). 


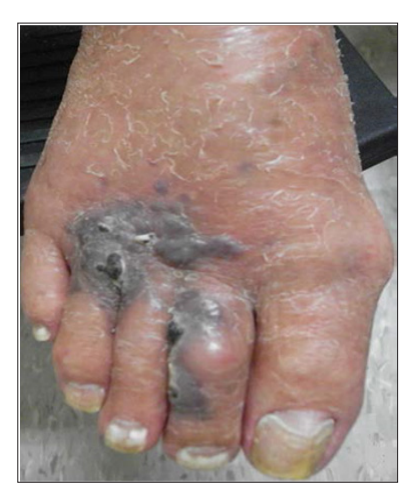

Figure 1b: Kaposi's sarcoma aggravated after two months of TB treatment.

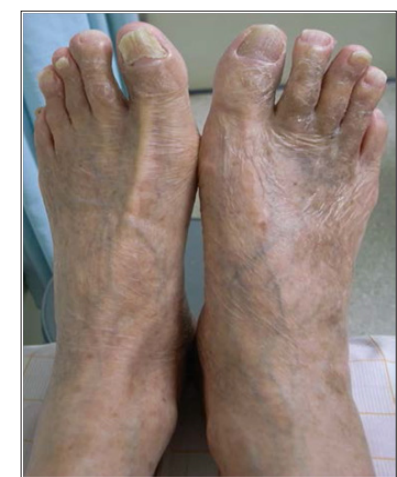

Figure 1c: Right foot Kaposi's sarcoma regressed after treatment.

\section{Discussion}

The etiology of Kaposi's sarcoma is complex and multifactorial. Reported epidemiologic data has revealed that KS has been seen in certain diseases, such as HIV infection, cytomegalovirus, hepatitis B virus, HTLV-1 (Human T-lymphotropic virus type 1), immune suppressed individuals [1-5], lymphoma [6], organ-transplanted patients $[7,8]$, patients receiving long-term immunosuppressive therapy [9], but KS has rarely been reported in tuberculosis patients. HHV-8 has been strongly associated with a viral cofactor in the pathogenesis of KS [10]. And whereas there were reports of KS with tuberculosis in HIV-infected patients, there were only four cases of KS related to tuberculosis infections in HIV negative patients found in the literature since 1988 [11-14]. One case developed rapid-deteriorating orificial tuberculosis concomitant with Kaposi's sarcoma and resulted in a fatal outcome [13].

Another case had multiple-drug resistant tuberculosis with a 2-year course of anti-tuberculosis treatment, and his KS regressed after 8 weeks' treatment of anti-tuberculosis, and for this reason, KS secondary to pulmonary tuberculosis was postulated [14]. In our case, we gave him 1 year of anti-TB treatment because of his disseminated tuberculosis involving the oral cavity and joint, and his KS regressed after anti-TB therapy without chemotherapy, radiotherapy or surgery. To our knowledge, this is the first case with TB of the oral cavity and right knee, with KS that survived over 5 years without chemotherapy and responded to anti-TB drug therapy only.
Regarding the treatment of KS, the major goals are symptomatic palliation, prevention of disease progression, and shrinkage of tumor to alleviate edema, organ compromise, and psychological stress [15]. In the study of the treatment of AIDS-associated KS, a combination ART (anti-retroviral therapy) is recommended for virtually all patients with AIDS-related KS, and may be the only therapy required in the absence of specific indications for chemotherapy $[16,17]$. The combination ART only in the patients with AIDS-associated KS can achieve a $20 \%$ to $39 \%$ response rate for KS $[16,17]$.

The incidence rate of TB infection in Taiwan was 62.0-74.6 (per 100,000 population) in the period of 2002-2008 [18], and the TB incidence with mortality rates was higher in southern regions than in the northern ones [19]. Thus, patients with KS should pay more attention to being observant of coexisting diseases, such as tuberculosis, especially in a high TB incidence area. Since there are limited case reports regarding the treatment of TB coinfected with KS, we presented this case with KS possibly secondary to TB, which regressed completely under anti-TB therapy only, without institution of any chemotherapy.

\section{References}

1. Moore PS, Chang Y (1995) Detection of herpes virus-like sequences in Kaposi's sarcoma in patients with and without HIV infection. N Engl J Med 332: 1181-1185.

2. Boshoff C, Shutz TF, Kennedy MM (1995) Kaposi's sarcoma- associated herpesvirus infects endothelial and spindle cells. Nature Med 1: 12741278.

3. Fenoglio CM, Oster MW, Lo Gerfo P (1982) Kaposi's sarcoma following chemotherapy for testicular cancer in a homosexual man - demonstration of CMV RNA in sarcomacells. Hum Pathol 13: 995-999.

4. Siddiqui A (1983) Hepatitis B virus DNA in Kaposi's sarcoma. Proc Natl Acad Sci USA 80: 4861-4864.

5. Veyssier-Belot C, Couderc LJ, Desgranges C (1990) Kaposi's sarcoma and HTLV-I infection lancet 336: 575.

6. Boshoff C, Whitby D, Talbot S, Weiss RA (2013) Etiology of AIDS- related Kaposi's sarcoma and lymphoma. Oral Dis 3: S129-S132.

7. Penn I (1987) Cancer following cyclosporin therapy. Transplantation 43: 32-35.

8. Boshoff C, Weiss RA (1998) Kaposi's sarcoma-associated herpes-virus. Adv Cancer Res 75: 57-86.

9. Penn I (1991) The changing pattern of posttransplant malignancies. Transplant Proc 23: 1101-1103.

10. Klepp O, Dahl O, Stenwig JT (1978) Association of Kaposi's sarcoma and prior immunosuppressive therapy: a 5-year material of Kaposi's sarcoma in Norway. Cancer 42: 2626-2630.

11. Konstantinov GS (1988) Kaposi's sarcoma combined with generalized miliary tuberculosis. Arkh Pathol 50: 58-61.

12. Castro A, Pedreira J, Soriano V (1992) Kaposi's sarcoma and disseminated tuberculosis in HIV-negative individual. Lancet 339: 868.

13. Chen YJ, Shieh P, Shen L (2000) Orificial tuberculosis and Kaposi sarcoma in an HIV-negative individual. Clin Exp Dermatol 25: 393-394.

14.Guler ZM, Kanbay A, Ciftci B (2005) Kaposi Sarcoma Secondary to Pulmonary Tuberculosis: A Rare Case. South Med J 98: 933-934.

15. Dezube BJ, Pantanowitz L, Aboulafia DM (2004) Management of AIDSrelated Kaposi sarcoma: advances in target discovery and treatment. AIDS Read 14: 236. 
16. Mosam A, Shaik F, Uldrick TS (2012) A randomized controlled trial of highly active antiretroviral therapy versus highly active antiretroviral therapy and chemotherapy in therapy-naive patients with HIVassociated Kaposi sarcoma in South Africa. J Acquir Immune Defic Syndr 60: 150 .

17. Martin-Carbonero L, Barrios A, Saballs P (2004) Pegylated liposomal doxorubicin plus highly active antiretroviral therapy versus highly active antiretroviral therapy alone in HIV patients with Kaposi's sarcoma. AIDS 18: 1737.

18. Centers for Disease Control: Department of Health, Executive Yuan Taiwan. Taiwan tuberculosis incidence and mortality rate, 2002-2008

19. Hsueh PR, Liu YC, So J, Liu CY, Yang PC, Luh KT (2006) Mycobacterium tuberculosis in Taiwan. J Infect 52:77-85.

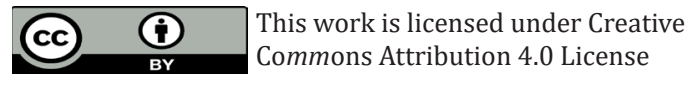

Submission Link: https://biomedres.us/submit-manuscript.php

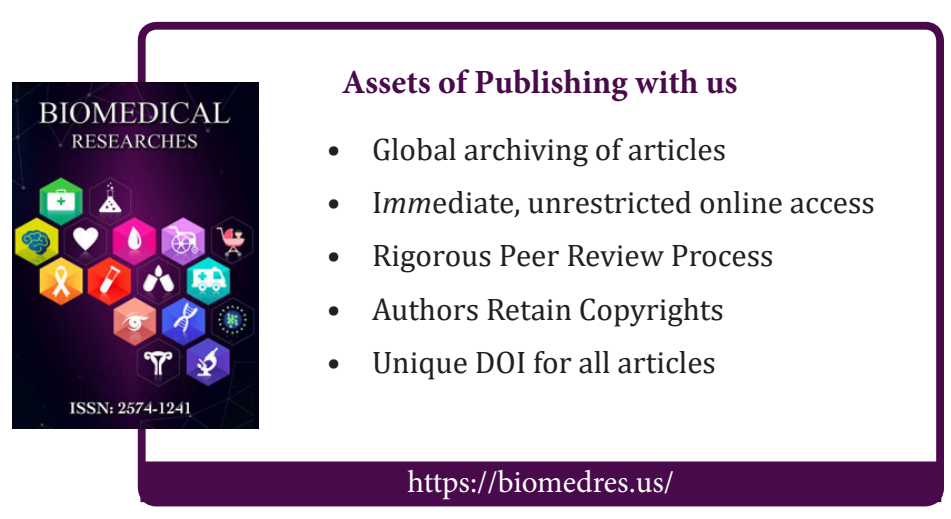

